\title{
Resonant Laser-Plasma Electron Acceleration
}

\author{
D. Umstadter ${ }^{1}$, E. Esarey ${ }^{2}$ and J. Kim ${ }^{1}$ \\ ${ }^{1}$ Center for Ultrafast Optical Science, University of Michigan, Ann Arbor, MI 48109 \\ ${ }^{2}$ Beam Physics Branch, Naval Research Laboratory, Washington, DC 20375
}

The generation of large-amplitude, relativistic plasma waves is a subject of much current interest because of its potential use for ultrahigh gradient electron acceleration. There are two major types of laser-driven, plasma-based accelerators: the plasma beatwave accelerator (PBWA) [1] and the laser wakefield accelerator (LWFA) [2]. While conventional rf-driven accelerators are limited to fields $\leq 1 \mathrm{MV} / \mathrm{cm}$, plasma accelerators have been shown experimentally to support gradients $\sim 10 \mathrm{MV} / \mathrm{cm}[3]$. The maximum axial electric field of a relativistic plasma wave, as predicted by $1 \mathrm{D}$ cold fluid theory, is the "wave-breaking" field, $E_{W B}=\left(m_{e} c \omega_{p} / e\right) \sqrt{2\left(\gamma_{p}-1\right)}$, which can exceed $1 \mathrm{GV} / \mathrm{cm}$, where $\omega_{p}=\left(4 \pi e^{2} n_{e 0} / m_{e}\right)^{1 / 2}$ is the electron plasma frequency, $n_{e 0}$ is the ambient electron density, $\gamma_{p}=\left(1-v_{p}^{2} / c^{2}\right)^{-1 / 2}$, and $v_{p}$ is the phase velocity of the plasma wave.

In the PBWA, two laser beams of frequencies $\omega$ and $\omega-\omega_{p}$ are optically mixed in a plasma to produce a laser beatwave, in effect a train of equally spaced pulses, which "resonantly" excites a large-amplitude plasma wave. A fundamental limitation to the plasma-wave amplitude in the PBWA is resonant detuning. As the plasma-wave amplitude grows, nonlinear effects cause the resonant frequency to shift away from $\omega_{p}$, which leads to saturation and thus limits the plasma-wave amplitude. In the LWFA, a single, intense, short laser pulse [4] drives a plasma wave "wakefield." The maximum plasma-wave amplitude results when $\tau \sim 2 \pi / \omega_{p}$, where $\tau$ is the laser pulse width, which translates into a "resonant density," since $\omega_{p} \sim n_{e 0}^{1 / 2}$. Recently, the self-modulated LWFA has been suggested [5], [6]. Here, a single laser pulse is incident on a plasma with a density that is higher than the "resonant density." Due to a self-modulation instability, the pulse breaks up into multiple pulses, each of which are "resonant." Although higher plasma densities and the multiple-pulse structure lead to higher wakefield amplitudes, both high plasma densities and high laser intensities are difficult to achieve simultaneously due to plasma defocusing, and electron acceleration is limited by phase detuning, i.e., accelerated electrons (with $v \rightarrow c$ ) outrun the plasma wave (with $v_{p} \simeq v_{g}<c$ ). The maximum energy gain, $\Delta W_{\text {max }}$, of an electron in a $1 \mathrm{D}$ sinusoidal plasma wave of amplitude $E_{z}=\epsilon E_{W B}$ is $\Delta W_{\max } \simeq 4 e c \epsilon E_{W B} \gamma_{p}^{2} / \omega_{p}$, where $\epsilon \leq 1$ is a constant. Since $\gamma_{p} \simeq \gamma_{g} \simeq \omega / \omega_{p} \gg 1$, $\Delta W_{\max } \simeq \epsilon m_{e} c^{2}\left(2 \gamma_{g}\right)^{5 / 2}$. For example, $\Delta W_{\max } \simeq 4.5 \mathrm{GeV}$, assuming a laser wavelength of $\lambda \simeq 2 \pi c / \omega=1 \mu \mathrm{m}, n_{e 0}=10^{18} \mathrm{~cm}^{-3}\left(E_{W B}=7.8 \mathrm{GV} / \mathrm{cm}\right)$ and $\epsilon=25 \%$. Hence, at the high densities required either for self-modulation or for the use of an ultrashort pulse in the standard LWFA, $\gamma_{g}$ is relatively low and acceleration is limited, $\Delta W \sim \epsilon n_{e 0}^{-5 / 4}$.

We propose an alternative concept, the resonant laser-plasma accelerator (RLPA), which combines the virtues of, but has certain advantages over, both the PBWA and the LWFA. In the RLPA, a series of laser pulses is utilized in which the length of the pulses, and the spacing between them, is varied in an optimized manner such that the maximum wakefield amplitude is achieved. The RLPA has the following advantages: (i) since a series of pulses is used, larger amplitude plasma waves can be generated than by the use of a single pulse with the same total energy, (ii) since the spacing between the 


\section{Resonant Laser-Plasma Electron}

pulses is varied, resonance with the nonlinear plasma wave can be maintained, (iii) lower plasma densities can be used, thus avoiding electron phase detuning, and (iv) lower peak laser intensities can be used, thus allowing for higher energy extraction from the laser system and a reduction of laser-plasma instabilities. A secondary pulse to enhance or diminish the wakefield has been suggested before [7], but a train of an arbitrary number of realistically shaped pulses has not been analyzed and optimized.

Just a few optimized square pulses are far more efficient than a single pulse. For example, at $n_{e 0}=10^{15} \mathrm{~cm}^{-3}\left(\lambda=1 \mu \mathrm{m}, \gamma_{g} \simeq 10^{3}, E_{W B} \simeq 1.4 \mathrm{GV} / \mathrm{cm}\right)$, three square pulses can be used with an intensity $I=2.7 \times 10^{18} \mathrm{~W} / \mathrm{cm}^{2} /$ pulse $\left(a_{0}^{2}=1\right)$ and a total pulse train energy of $I \tau_{\text {tot }}=20 \mathrm{MJ} / \mathrm{cm}^{2}$ to produce $E_{z}=0.1 \mathrm{GV} / \mathrm{cm}$. Here, $\tau_{\text {tot }}$ is the sum of the pulse durations in the train and $2.7 a_{0}^{2} \simeq 10^{-18} \lambda^{2}[\mu \mathrm{m}] I\left[\mathrm{~W} / \mathrm{cm}^{2}\right]$. A single pulse at $n_{e 0}=10^{15} \mathrm{~cm}^{-3}$ requires $I=3 \times 10^{19} \mathrm{~W} / \mathrm{cm}^{2}\left(a_{0}^{2}=11\right)$ or a pulse energy six times greater $\left(I \tau_{\text {tot }}=120 \mathrm{MJ} / \mathrm{cm}^{2}\right)$ to produce this same $E_{z}$.

There are several ways of producing the required pulse train in practice. The first is to use Fourier filtering. In this case, a mask placed in the pulse stretcher of a CPA system is used to modulate the phase and/or frequency of the beam in such a way that, when it is recompressed, a series of pulses with arbitrary spacings and widths will be produced [8]. The minimum rise time of each individual pulse is still governed by the gain bandwidth of the amplifiers. The second possibility is to use several separate compressors with adjustable lengths and delays.

This work was supported by NSF and DoE.

\section{References}

[1] T. Tajima and J.M. Dawson, Phys. Rev. Lett. 43, 267 (1979).

[2] L.M. Gorbunov and V.I. Kirsanov, Sov. Phys. JETP 66, 290 (1987); P. Sprangle, et al., Appl. Phys. Lett. 53, 2146 (1988).

[3] C. Clayton, et al., Phys. Rev. Lett. 54, 2343 (1985); Y. Kitigawa, et al., Phys. Rev. Lett. 68, 48 (1992); F. Amiranoff, et al., Phys. Rev. Lett. 68, 3710 (1992).

[4] P. Maine, et al., IEEE J. Quant. Electron. 24, 398 (1988); G. Mourou and D. Umstadter, Phys. Fluids B 4, 2315 (1992).

[5] P. Sprangle, et al., Phys. Rev. Lett. 69, 2200 (1992); E. Esarey, et al., Phys. Fluids B 5, 2690 (1993); J. Krall, et al., Phys. Rev. E 48, 2157 (1993).

[6] N.E. Andreev, et al., JETP Lett. 55, 571 (1992); T. Antonsen, Jr. and P. Mora, Phys. Rev. Lett. 69, 2204 (1992).

[7] V.I. Berezhiani and I.G. Murusidze, Physica Scripta 45, 87 (1991); K. Nakajima, Coherent Radiation Generation and Particle Acceleration, ed. by A. Prokhorov (Amer. Inst. Phys., NY, 1992), p. 436.

[8] A.M. Weiner, et al., IEEE J. Quant. Electron. 28, 908 (1992). 